\title{
Developing Consilience Medicine: Positron Emission Tomography Scan and Transcriptomics in Pancreatic Cancer
}

\author{
Seong Hun Kim ${ }^{1,2}$ and Seung OK Lee ${ }^{1,2}$ \\ ${ }^{1}$ Department of Internal Medicine, Chonbuk National University Hospital, Chonbuk National University Medical School, and ${ }^{2}$ Biomedical \\ Research Institute, Chonbuk National University Hospital, Jeonju, Korea
}

See "Preoperative Metabolic Tumor Volume ${ }_{2.5}$ Associated with Early Systemic Metastasis in Resected Pancreatic Cancer: A Transcriptome-Wide Analysis" by Sung Hwan Lee, et al. on page 356, Vol. 13, No. 3, 2019

Consilience medicine is the merging of biology, physics, chemistry, physiology, biochemistry, and molecular genetics for studying diseases. Nowadays, there is increased need for consilience medicine for precision or personalized medicine, which is an approach to patient care that allows doctors to select the most suitable treatments for patients based on their genetic background. In this pilot translational study, link of positron emission tomography/computed tomography (PET/CT) scan results and metabolic phenotype which are produced by transcriptome-wide analysis was tried with regard to pancreatic cancer prognosis. ${ }^{1}$ Studies that marry clinical data and genetic, epigenetic or transcriptomic data like one in this paper should be done more for the advancement of precision medicine in the future.

Although its effect on the staging of pancreatic cancer remains indeterminate, PET can be used as a valuable diagnostic and predictive tool. In meta-analysis, the pooled sensitivity and specificity of PET in pancreatic cancer diagnosis are known to be 0.91 and 0.81 , respectively. In prognosis analysis, significant difference in overall survival was observed between high and low standardized uptake value groups (hazard ratio, 2.39). ${ }^{2}$ PET/ CT outperforms all other modes of imaging in the detection of distant metastases, allowing more accurate staging, despite its limitations in loco-regional assessment of lymphadenopathy and vascular invasion which can be overcome by the CT component of the evaluation. ${ }^{3}$

The standard uptake value (SUV) is a semiquantitative parameter usually used for the interpretation of PET images. The maximum standard uptake value $\left(\mathrm{SUV}_{\max }\right)$ which corresponds to the maximal pixel value in the tumor can be calculated from one-pixel region of interest. It has been a very common parameter for cancer evaluation due to its ease of use. $\mathrm{SUV}_{\max }$ has a correlation with survival and higher predictive value for recurrence in various malignancies. ${ }^{4}$ However, $\mathrm{SUV}_{\max }$ could be observer-dependent depending on the region of interest usually determined by an individual examiner. However, it is difficult to reflect the accurate metabolic activity in heterogeneous tumors using a single pixel. In order to resolve these challenges posed by $\mathrm{SUV}_{\text {max }}$, several volume-based PET parameters such as metabolic tumor volume (MTV) and total lesion glycolysis (TLG) have been introduced to accurately estimate the metabolic activity of an entire tumor. The MTV, defined as the volume of FDG activity in a tumor assessed by automated volume of interest delineation, and TLG, defined as the product of MTV and $\mathrm{SUV}_{\text {mean }}$, may be more representative of the tumor heterogeneity. MTV values could be expressed as MTV25\%, MTV42\%, MTV50\% and MTV75\%, which are defined tumor volumes with an absolute threshold of 25\%, 42\%, 50\% and 75\% of the histogram of $\mathrm{SUV}_{\max }$, respectively. If segmentation was at a fixed threshold (SUV >2.5), MTV 2.5 is defined as tumor volume with SUV > 2.5, whereas TLG $_{2.5}$ was defined as the product of SUV $_{\text {mean }}$ and $\mathrm{MTV}_{2.5}$. In the results of studies evaluating the role of MTV/ TLG and SUV $_{\text {max }}$, MTV/TLG was more predictive for clinical outcome, with a higher predictive value as compared to $\mathrm{SUV}_{\max }{ }^{4-6}$ Similarly, in this study, higher MTV $_{2.5}$ values $\left(\mathrm{MTV}_{2.5} \geq 4.5\right)$ were associated with multiple lymph node metastasis and poor specific survival of patients compared to patients having $\mathrm{MTV}_{2.5}$ $<4.5$. Interestingly, the group of patients with $\mathrm{MTV}_{2.5} \geq 4.5$ and

Correspondence to: Seung Ok Lee (https://orcid.org/0000-0003-0243-215X)

Department of Internal Medicine, Chonbuk National University Hospital, Chonbuk National University Medical School, 20 Geonji-ro, Deokjin-gu, Jeonju 54907, Korea

Tel: +82-63-250-1289, Fax: +82-63-255-1609, E-mail: solee@jbnu.ac.kr pISSN 1976-2283 eISSN 2005-1212 https://doi.org/10.5009/gnl19116

(a) This is an Open Access article distributed under the terms of the Creative Commons Attribution Non-Commercial License (http://creativecommons.org/licenses/by-nc/4.0) which permits unrestricted non-commercial use, distribution, and reproduction in any medium, provided the original work is properly cited. 
subsequently postoperative adjuvant chemotherapy showed better survival outcomes than patients with $\mathrm{MTV}_{2.5} \geq 4.5$ lacking adjuvant treatment in resected pT3 pancreatic cancer. This result suggests that cancers with higher metabolic activity are more sensitive to chemotherapy.

Genetics is a biologic branch that is tasked with the study of genes, genetic variation and heredity that relates to DNA sequences. However, genetics cannot explain all physiologic and pathologic processes because the gene expression patterns are quite different in various cell types and at different point in time even though they have similar DNA sequences. To resolve these problems, other branches of biologics such as epigenetics, transcriptomics and proteomics have been actively studied and developed. Epigenetics is study of relevant functionally changes of the genome such changes are DNA methylation and histone modification that do not involve a change in the nucleotide sequence itself. Transcriptomics is the study of transcriptome using high-throughput methods, such as microarray or RNAseq analysis. Transcriptomes are complete set of RNA transcripts which are differentially expressed in distinct cell populations under specific circumstances or time points. ${ }^{7}$

Comparison of transcriptomes allows for the identification of genes that are differentially expressed in distinct cell populations, in the case of any pathologic change or response to different treatments. Similarly, in pancreatic cancer, key regulators such as HNF1A can be found using comparative analysis of transcriptomes in normal and tumor tissue samples. ${ }^{8}$ In this study, a transcriptome-wide analysis was performed on a patients' tumor tissue to reveal the differences in genetic expression on metabolic phenotypes identified on PET scans preoperatively. The analysis revealed that tumors with $\operatorname{MTV}_{2.5} \geq 4.5$ showed broad enhancement of cancer-related gene expression, especially in genes related to cancer progression and metastasis compared to the low group $\left(\mathrm{MTV}_{2.5}<4.5\right)$. This paper appeared to be a typical example of a study showing significant results from the linkage of the image analysis to molecular work data.

In conclusion, this pilot translational study revealed the prognostic value of metabolic phenotype that can be identified through preoperative PET scan and the potential correlation between gene expressions related to aggressive tumor behavior and high metabolism in pancreatic cancer. If additional studies using a larger number of patients were done, these kinds of results could be used for precision or personalized medicine such as neoadjuvant treatment for patients with unresectable and borderline resectable pancreatic cancers, or adjuvant treatment for resectable cancers.

\section{CONFLICTS OF INTEREST}

No potential conflict of interest relevant to this article was reported.

\section{REFERENCES}

1. Lee SH, Hwang HK, LEE WJ, Yun M, Kang CM. Preoperative metabolic tumor volume2.5 associated with early systemic metastasis in resected pancreatic cancer: a transcriptome-wide analysis. Gut Liver 2019;13:356-365.

2. Wang Z, Chen JQ, Liu JL, Qin XG, Huang Y. FDG-PET in diagnosis, staging and prognosis of pancreatic carcinoma: a metaanalysis. World J Gastroenterol 2013;19:4808-4817.

3. Jha P, Bijan B. PET/CT for pancreatic malignancy: potential and pitfalls. J Nucl Med Technol 2015;43:92-97.

4. Castelli J, De Bari B, Depeursinge A, et al. Overview of the predictive value of quantitative 18 FDG PET in head and neck cancer treated with chemoradiotherapy. Crit Rev Oncol Hematol 2016;108:40-51.

5. Kang CM, Lee SH, Hwang HK, Yun M, Lee WJ. Preoperative volume-based PET parameter, MTV2.5, as a potential surrogate marker for tumor biology and recurrence in resected pancreatic cancer. Medicine (Baltimore) 2016;95:e2595.

6. Ma W, Wang M, Li X, et al. Quantitative (18)F-FDG PET analysis in survival rate prediction of patients with non-small cell lung cancer. Oncol Lett 2018;16:4129-4136.

7. Lowe R, Shirley N, Bleackley M, Dolan S, Shafee T. Transcriptomics technologies. PLoS Comput Biol 2017;13:e1005457.

8. Hoskins JW, Jia J, Flandez M, et al. Transcriptome analysis of pancreatic cancer reveals a tumor suppressor function for HNF1A. Carcinogenesis 2014;35:2670-2678. 